\title{
Safety of hormone replacement therapy after mastectomy
}

\section{Clinical question}

After mastectomy, chemotherapy and radiotherapy for high-grade ductal carcinoma with nodal involvement and lymphatic infiltration, a 48-year-old woman had premature menopause, with symptoms of loss of libido. Her general practitioner wanted to know how safe and effective hormone replacement therapy (HRT) would be for this patient.

\section{Search question}

The revised question was: "Does HRT increase the likelihood of recurrence in a patient previously diagnosed with breast cancer?"

The ideal study to answer this question would be a randomised controlled trial of HRT versus placebo comparing outcomes of mortality and recurrence in women previously treated for breast cancer or high-grade ductal carcinoma of the breast.

\section{Search}

We searched two online databases: PubMed Clinical Queries < http://www.ncbi.nlm.nih.gov/entrez/query/static/ clinical.html> and the Cochrane Library, using the search terms "hormone replacement therapy", "breast cancer", "breast neoplasm", "mastectomy" and "libido".

\section{Summary of findings}

No ideal studies have been published. However, five observational studies (three cohort and two case-control) were identified that reported the occurrence of adverse events after administration of HRT in women previously treated for breast cancer.

at Two of the cohort studies were prospective studies of 24 and 25 women who had received HRT after treatment for breast cancer. ${ }^{1,2}$ No recurrences were reported in the 24 women observed over a period of $24-44$ months; ${ }^{1}$ and three recurrences occurred in the 25 women after a mean follow-up of 30.4 months (survival rate, 96\%). ${ }^{2}$ In the retrospective cohort study, seven recurrences occurred in 77 women; ${ }^{3}$ the average interval from diagnosis to relapse was 45.3 months.

at A case-control study was conducted in a subset of the 77 patients in the cohort study mentioned above. Forty-one patients who received HRT were matched with 82 controls not receiving HRT. There were no significant differences in disease-free times and survival times between the two groups. ${ }^{4}$
The second case-control study of women after primary surgical treatment, in which 21 women received HRT and 42 matched controls did not, reported relapse in four patients in the treatment group (19\%) and five in the control group (11\%). The authors estimated the risk of relapse of breast cancer among women who had received HRT for a mean of 28 months compared with the control group (odds ratio [OR], $1.74 ; 95 \% \mathrm{CI}, 0.34$ 8.88), and among those who had received HRT for less than 24 months (OR, 0.65; 95\% CI, 0.02-7.85). ${ }^{5}$

\section{Comment}

In the studies identified in this search, there was little evidence of increased recurrence of breast cancer with the use of HRT in patients previously diagnosed with breast cancer. However, these studies involved small numbers of patients, the duration of follow-up was not long, and there was potential for bias. Caution should prevail until appropriate clinical trials are conducted.

\section{Outcome}

After weighing up the evidence against the benefits of menopause symptom control, the general practitioner continued to prescribe HRT for this patient.

Christopher B Del Mar

Professor

Paul P Glasziou

Professor

Anneliese B Spinks

Research Officer

Sharon L Sanders

Research Officer

Deborah J Hilton

Research Officer

Centre for General Practice, Medical School University of Queensland, Herston, QLD

c.delmar@cgp.uq.edu.au

\section{References}

1. Guidozzi F. Estrogen replacement therapy in breast cancer survivors. Int J Gynaecol Obstet 1999; 64: 59-63.

2. Wile AG, Opfell RW, Margileth DA. Hormone replacement therapy in previously treated breast cancer patients. Am J Surg 1993; 165: 372-375

3. DiSaia PJ, Grosen EA, Odicino F, et al. Replacement therapy for breast cancer survivors. A pilot study. Cancer 1995; 76: 2075-2078.

4. DiSaia PJ, Grosen EA, Kurosaki T, et al. Hormone replacement therapy in breast cancer survivors: a cohort study. Am J Obstet Gynecol 1996; 164: 1494-1498.

5. Ursic-Vrscaj M, Bebar S. A case-control study of hormone replacement therapy after primary surgical breast cancer treatment. Eur J Surg Oncol 1999; 25: 146151 